\title{
Complexity Quantification for Overhead Transmission Line Emergency Repair Scheme via a Graph Entropy Method Improved with Petri Net and AHP Weighting Method
}

\author{
Jing Zhou, ${ }^{1,2}$ Cunbin Li, ${ }^{2}$ Weihua Shen, ${ }^{3}$ and Ye Zhang ${ }^{1}$ \\ ${ }^{1}$ School of Control and Computer Engineering, North China Electric Power University, Beijing 102206, China \\ ${ }^{2}$ School of Economics and Management, North China Electric Power University, Beijing 102206, China \\ ${ }^{3}$ State Grid Shanxi Electric Power Company, Taiyuan 030001, China \\ Correspondence should be addressed to Jing Zhou; zhoujingncepu@163.com
}

Received 22 September 2014; Revised 4 December 2014; Accepted 9 December 2014; Published 21 December 2014

Academic Editor: Muhammad Naveed Iqbal

Copyright ( 2014 Jing Zhou et al. This is an open access article distributed under the Creative Commons Attribution License, which permits unrestricted use, distribution, and reproduction in any medium, provided the original work is properly cited.

\begin{abstract}
According to the characteristics of emergency repair in overhead transmission line accidents, a complexity quantification method for emergency repair scheme is proposed based on the entropy method in software engineering, which is improved by using group AHP (analytical hierarchical process) method and Petri net. Firstly, information structure chart model and process control flowchart model could be built by Petri net. Then impact factors on complexity of emergency repair scheme could be quantified into corresponding entropy values, respectively. Finally, by using group AHP method, weight coefficient of each entropy value would be given before calculating the overall entropy value for the whole emergency repair scheme. By comparing group AHP weighting method with average weighting method, experiment results for the former showed a stronger correlation between quantified entropy values of complexity and the actual consumed time in repair, which indicates that this new method is more valid.
\end{abstract}

\section{Introduction}

Electric power industry is vital to China's economy and power grid plays a very important role in transferring electricity. The faults of overhead transmission lines are diverse, the locations of faults are stochastic, and circumstances of faults are complex $[1,2]$ so that the emergency repair of overhead transmission lines is a sophisticated task. According to the initial assessment, emergency personnel could figure out an emergency repair scheme. However, the rationality and feasibility of the scheme are all related to the complexity of task. Therefore, to precisely quantify and comprehensively analyze the complexity of emergency repair task has been a hot research topic [3].

So far, with regard to complexity quantification on emergency repair scheme, most overseas and domestic scholars have been focusing on factors which influence the complexity and graphical modeling methods. In the literature [4], quantified impact factors on emergency accident were analyzed for nuclear power plants. Besides three basic impact factors in software engineering, the abstract hierarchical architecture of task and the concept of engineering judgment were proposed. Finally the validity of impact factors is tested using assessment results. In the literature [5], a graph entropy method was applied in emergency maintenance for marine power facilities, which verified the correlation between MTC (maintenance task complexity) and the consumed time in maintenance. In the literature [6], a graphical modeling method for the operational processes was improved. By using Petri net, the model of operational processes was built, from which information structure chart and action structure chart were extracted. However, selected operational objects were only partial of the whole task so that the quantification in that method was limited.

In software engineering, software complexity measure includes two kinds of methods. The first is based on source code quantification and the second is based on structure and chart. In the literature [7], entropy method was selected to 
assess the complexity of marine power facility operation after similar characteristics were compared between software complexity and emergency process complexity. Since the complexity of source code resembles the one of logic structure and program maintenance which requires amount of source code knowledge resembles emergency maintenance that requires amount of system information and background knowledge, entropy method can reflect not only the complexity of information but also the complexity of logic. In the literature [8], graph entropy method was proposed as a way to quantify the complexity based on information structure chart and process control flowchart.

To improve the accuracy of complexity quantification in the sophisticated power grid, in this paper, Petri net will be used to model an intact emergency repair task based on graph entropy method. To improve the design of weights in computation of complexity, group AHP method is applied to set weights based on experts' advice so that the design of weights is more pertinent and its subjective errors could be also reduced greatly.

\section{Complexity Quantification Method}

2.1. Extraction of Impact Factors on the Complexity Quantification of Emergency Repair Task. Emergency repair tasks along transmission lines are always characterized by the urgency of the time, the randomness in the form of damage, the flexibility of the way of repair, and the diversity of the recovery phase. Therefore, in the emergency repair process, faults should be eliminated as soon as possible by making the most of resources and techniques so as to diminish economic loss and reduce threats to security of power grid. Features of facilities, the damaged condition, the composition of the needed equipment and professionals, and the difficulties to assemble or disassemble components should be all taken into account. Besides, the allowed time of repair, the way of locating the damage, the isolation level after the damage, and the requirement on tools should be also considered.

To sum up, complexity quantification in emergency repair of transmission lines is a comprehensive measure about needed workload, resource, consumed time, and logic structure when damages to facilities are caused by accidents and emergency repair is necessary. The impact factors on complexity quantification could be sorted into 3 kinds: information complexity (IC), logic structure complexity (LC), and scale complexity (SC) of operation in emergency rescue and repair.

2.2. Graph Entropy Modeling. Graph entropy method is a way to quantify complexity based on information structure chart and process control flowchart. By establishing graph entropy model, the abstract process information can be displayed. There are two main ways of quantification: (1) chromatic information content, that is, the first-order entropy; (2) structural information content, that is, the second-order entropy.

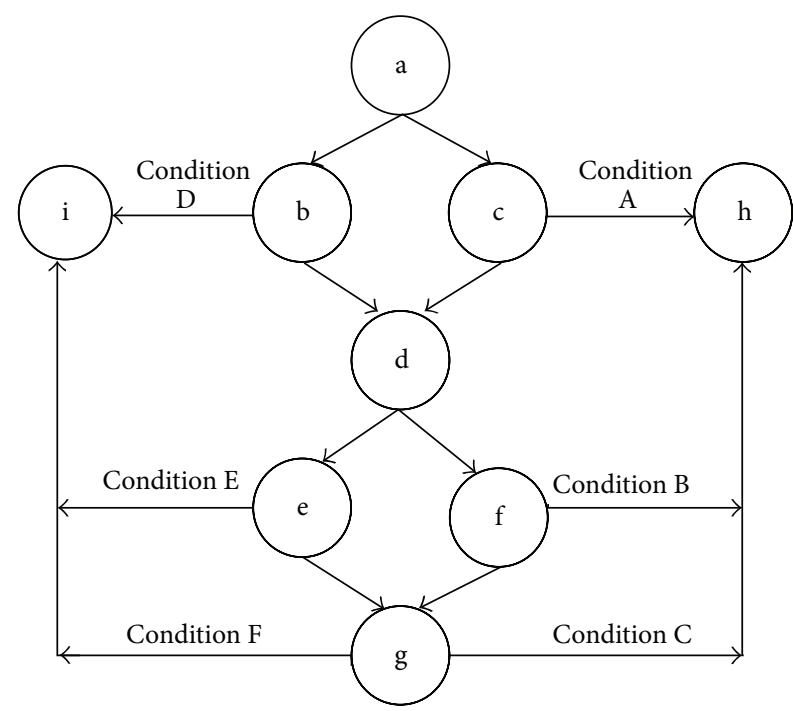

Figure 1: Process control flowchart model $G$.

(1) The formula to calculate the graph entropy value is shown below:

$$
\begin{gathered}
\text { Entropy }=H=P=-\sum_{i=1}^{h} p\left(A_{i}\right) \log _{2} p\left(A_{i}\right), \\
p\left(A_{i}\right)=\frac{m}{n},
\end{gathered}
$$

where $A_{i}$ is a category defined according to the structure chart; $h$ is the total number of categories; $p\left(A_{i}\right)$ is the assessed probability of being in category $A_{i} ; m$ is the number of nodes in category $A_{i}$; and $n$ is the total number of nodes.

(2) Application of graph entropy method will be illustrated based on the process control flowchart model $G$ (Figure 1).

Here, operation $\mathrm{h}$ and operation $\mathrm{i}$ are the same-type operations, but their results are opposite. In this model, logic complexity (LC) can be quantified by calculating the firstorder entropy, and scale complexity (SC) can be quantified by calculating the second-order entropy. While calculating the first-order entropy, those nodes with the same number of inputs and outputs in control flowchart are classified into the same category. While calculating the second-order entropy, adjacent nodes with same number and same type within an arc distance in control flowchart are classified into the same category.

Based on the above rules of classification, nodes for model $G$ are classified and listed in Table 1 .

By taking the calculation of the first-order entropy in model $G$ as an example, nodes for the first-order entropy are classified into four categories as $\{a\},\{b, c, e, f\},\{d, g\}$, and $\{\mathrm{h}, \mathrm{i}\}$. $H$ represents entropy value of each impact factor. The probabilities for each category are 1/9, 4/9, 2/9, and 2/9, 
TABLE 1: Classification and calculation for the first- and secondorder entropy of model $G$.

\begin{tabular}{|c|c|c|c|c|c|}
\hline \multirow[t]{2}{*}{ Category } & \multicolumn{3}{|c|}{$\begin{array}{l}\text { The first-order entropy } \\
\text { for model } G\end{array}$} & \multicolumn{2}{|c|}{$\begin{array}{l}\text { The second-order } \\
\text { entropy for } \\
\text { model } G\end{array}$} \\
\hline & Input & Output & Node & Node & $\begin{array}{c}\text { Neighbor } \\
\text { node }\end{array}$ \\
\hline 1 & 0 & 2 & $\mathrm{a}$ & a & $b, c$ \\
\hline 2 & 1 & 2 & $\begin{array}{l}b, c, e, \\
\text { and } f\end{array}$ & b, c & $\begin{array}{l}\text { a, } \mathrm{d} \text {, and } \mathrm{h} \\
\quad \text { (or i) }\end{array}$ \\
\hline 3 & 2 & 2 & $\mathrm{~d}, \mathrm{~g}$ & $\mathrm{~d}$ & $\mathrm{~b}, \mathrm{c}, \mathrm{e}$, and $\mathrm{f}$ \\
\hline 4 & 3 & 0 & h, i & $e, f$ & $\begin{array}{l}\mathrm{d}, \mathrm{g} \text {, and } \mathrm{h} \\
\text { (or i) }\end{array}$ \\
\hline 5 & & & & g & $e, f, h$, and $i$ \\
\hline 6 & & & & $\mathrm{~h}$ & $\mathrm{c}, \mathrm{f}$, and $\mathrm{g}$ \\
\hline 7 & & & & $\mathrm{i}$ & $\mathrm{b}, \mathrm{e}$, and $\mathrm{g}$ \\
\hline $\begin{array}{l}\text { Entropy } \\
\text { value }\end{array}$ & & 1.8366 & & & 255 \\
\hline
\end{tabular}

respectively. According to formula (1), the first-order entropy for model $G$ is worked out as below:

$$
\begin{aligned}
G_{1}= & H_{G}^{1}=-\sum_{i=1}^{4} p\left(A_{i}\right) \log _{2} p\left(A_{i}\right) \\
= & -\left(\frac{1}{9} \log _{2} \frac{1}{9}\right)-\left(\frac{4}{9} \log _{2} \frac{4}{9}\right) \\
& -2\left(\frac{2}{9} \log _{2} \frac{2}{9}\right)=1.8366 .
\end{aligned}
$$

In the same way, the second-order entropy for model $G$ is worked out as follows:

$$
\begin{aligned}
G_{2} & =H_{G}^{2}=-\sum_{i=1}^{5} p\left(A_{i}\right) \log _{2} p\left(A_{i}\right) \\
& =-5\left(\frac{1}{9} \log _{2} \frac{1}{9}\right)-2\left(\frac{2}{9} \log _{2} \frac{2}{9}\right)=2.7255 .
\end{aligned}
$$

In a graph entropy model, a node represents a unit of information, and an arc represents the relationship between nodes. Data types of information include type $\mathrm{C}$ (character), type B (Boolean), type F (floating), and other customized types. Information complexity (IC) of maintenance task could be worked out by calculating the second-order entropy value of information structure chart model using graph entropy method [9].

Until now, complexity quantification methods for the three impact factors above have been all presented.

2.3. Process Control Graphical Modeling Based on Petri Net. Process control flowchart worked out by different graphical modeling methods will be in different structure, which will directly influence the quantification of task complexity. At present, there are mainly the following graphical modeling methods for process control flowchart: UML (unified modeling language), Petri net, and PERT chart. Among them, PERT

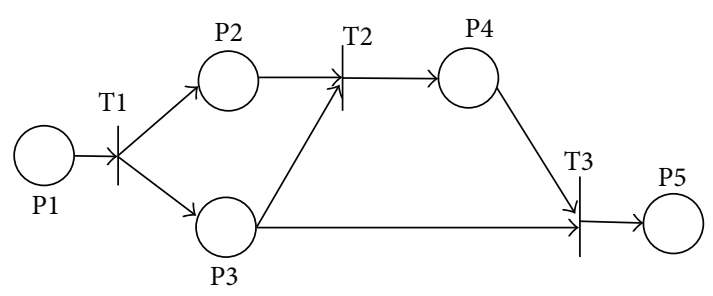

FIgure 2: Graph modeling in Petri net.

chart is mainly used to determine the vital path that influences the overall situation but could not reflect the amount of information needed in emergency maintenance task. The graphical modeling in UML is relatively simple, but it overemphasizes the logic of operation so that the needed information in emergency maintenance task is represented insufficiently [10]. Petri net not only could draw the logic structure chart with concepts of Library and Transition, but also could describe the amount of information and of emergency repair resource in the form of resource while the system changes; thus this method is preferred to the other two methods [11].

Petri net is a triple; that is, $N=(P, T, F)$. In Petri net, $P=\left\{p_{1}, p_{2}, \ldots, p_{h}\right\}$ is a set of Library, and the status of system is described by the elements of $p_{1} \cdots p_{h}$, respectively; $T=$ $\left\{t_{1}, t_{2}, \ldots, t_{k}\right\}$ is a set of Transition, and the change of system status is described by the elements of $t_{1} \cdots t_{k}$, respectively. $P$ and $T$ belong to different categories. $F=(P \times T) \cup(T \times P)$ is a set of input functions and output functions, which establishes a single-track connection from Library to Transition or from Transition to Library, where it is required that homogeneous elements cannot connect with each other [12]. A simple graphical modeling in Petri net is shown in Figure 2.

At first, information structure chart is built applying Petri net. Library in Petri net is a variable of information taken as the top node in the information structure chart and the type of the variable is taken as the bottom node. If a variable includes more than one resource, it should be connected with a variable pool and then each resource in the pool could be connected with the type of this variable. Besides, Petri net has a logic control structure itself so that it can be directly used as process control flowchart. After quantification of complexity for the three factors, the quantified value of overall complexity of emergency repair scheme can be calculated as below:

$$
\mathrm{CT}=\sqrt{\alpha(\mathrm{IC})^{2}+\beta(\mathrm{LC})^{2}+\gamma(\mathrm{SC})^{2}},
$$

where $\alpha, \beta$, and $\gamma$ are weights of different factors' complexity and CT is short for the complexity of task.

2.4. Weight Setting. Weights $(\alpha, \beta, \gamma)$ will be set by method of group AHP [13], and then the similarity can be determined based on experts' judgment on consistency. Next, weights should be recalculated in order to decrease subjective errors [14]. It is assumed that $n$ complexity factors will be assessed by $m$ experts; $A^{k}=\left(a_{i j}^{k}\right)_{n \times n}$ is the judgment matrix made by the expert $k . a_{i j}^{k}$ is the relative importance of complexity factor $i$ 
to factor $j$. Here are $0<a_{i j}^{k}<10$ and $a_{i j}^{k}=1 / a_{j i}^{k}$. After the judgment matrix is given, its consistency should be tested until it is satisfying, and then weights could be calculated [15]. The process is as follows.

Step 1. $m$ experts are classified into $m$ types; there are $Z=$ $\left\{Z_{1}, Z_{2}, \ldots, Z_{m}\right\}$ and $r=m$. Based on the judgment matrix, the standard matrix is expressed as $B^{k}=\left(b_{i}^{k}\right)_{n \times 1}$; herein,

$$
b_{i}^{k}=\frac{1}{n} \sum_{j=1}^{n} \frac{a_{i j}^{k}}{\sum_{i=1}^{n} a_{i j}^{k}} .
$$

Step 2. The similarity is assessed by calculating the vector angle $c_{p q}$. The larger the value of $c_{p q}$ is, the more similar the assessment result is:

$$
c_{p q}=\frac{B^{p} \cdot B^{q}}{\left|B^{p}\right| \times\left|B^{q}\right|} .
$$

Step 3. $p$ and $q$ are combined into type $m+1$; there are $Z=$ $\left\{Z_{1}, Z_{2}, \ldots, Z_{p-1}, Z_{p+1}, \ldots, Z_{q-1}, Z_{q+1}, \ldots, Z_{m}, Z_{m+1}\right\}, r=$ $r+1, c_{i, q+1}=\max \left\{c_{i s}, c_{i t}\right\}$, and $i \neq p$,q. If $r=2(m-1)$ is true, then the cluster chart is worked out and the process stops. Otherwise, this step is repeated.

It is assumed that there are $s$ types of experts and $\xi_{k}$ experts in type $k$, and then weight of each type of experts is as below:

$$
\alpha_{k}=\frac{\xi_{k}}{\sum_{i=1}^{s} \xi_{i}} .
$$

Via the equation below, the weight matrix $W$ could be worked out for each complexity impact factor:

$$
\min f(\omega)=\sum_{i=1}^{n} \sum_{j=1}^{n} \sum_{k=1}^{m} \alpha_{k}\left[\ln \left(a_{i j}^{k}\right)-\ln \left(\frac{\omega_{j}}{\omega_{i}}\right)\right]^{2} .
$$

If $\partial f(\theta) / \partial \theta_{j}=0$ is satisfied, then $W$ is calculated below:

$$
W=\left(\omega_{j}\right)_{n \times 1}=\left(\frac{\left[\prod_{i=1}^{n} \prod_{k=1}^{m} a_{i j}^{\alpha_{k}}\right]^{1 / n}}{\sum_{j=1}^{n}\left[\prod_{i=1}^{n} \prod_{k=1}^{m} a_{i j}^{\alpha_{k}}\right]^{1 / n}}\right)_{n \times 1} .
$$

\section{Case Study}

An overhead transmission line is out of order, and its hanging clamps need replacing. The information structure chart model for emergency repair task is shown in Figure 3.

The meanings of Library and Transition are shown below, and the process control flowchart is shown in Figure 4.

A1: the safety requirement is met at accident site.

A2: electroscope meets the requirement for voltage classes.

\begin{tabular}{|c|c|c|c|c|c|}
\hline \multirow{2}{*}{ Category } & \multicolumn{3}{|c|}{ First-order entropy of $G$} & \multicolumn{2}{|c|}{ Second-order entropy of $G$} \\
\hline & Node & Input & Output & Node & Neighbor node \\
\hline 1 & $\{\mathrm{~A} 1\}$ & 0 & 1 & $\begin{array}{c}\{\mathrm{A} 2, \mathrm{~A} 3 \\
\mathrm{A} 4\}\end{array}$ & $\{\mathrm{T} 1, \mathrm{~T} 2\}$ \\
\hline 2 & $\{\mathrm{~A} 10\}$ & 1 & 0 & $\{\mathrm{~A} 1\}$ & $\mathrm{T} 1$ \\
\hline 3 & $\begin{array}{l}\{\mathrm{A} 2 \text { to } \\
\mathrm{A} 9, \mathrm{~T} 3 \\
\text { to } \mathrm{T} 8\}\end{array}$ & 1 & 1 & $\{\mathrm{~T} 1\}$ & $\{\mathrm{A} 1, \mathrm{~A} 2, \mathrm{~A} 3, \mathrm{~A} 4\}$ \\
\hline 4 & $\{\mathrm{~B} 1\}$ & 2 & 2 & $\cdots$ & $\cdots$ \\
\hline 5 & $\{\mathrm{~T} 1\}$ & 1 & 3 & $\{\mathrm{~T} 8\}$ & $\{\mathrm{A} 9, \mathrm{~A} 10\}$ \\
\hline 6 & $\{\mathrm{~T} 2\}$ & 3 & 1 & $\{\mathrm{~A} 10\}$ & $\{\mathrm{T} 8\}$ \\
\hline
\end{tabular}

A3: the safety distance between operator and electroscope is more than $1.5 \mathrm{~m}$.
TABLE 2: Classification on the first- and the second-order entropy of process control flowchart.

A4: the insulation of extendible electroscope is long enough.

A5: there is no voltage in transmission lines and the specification of ground wire meets the requirement.

A6: ground wire has been connected, and transfer tackle has been fixed.

A7: two electricians have prepared for conductor backup protection and have been waiting on cross arms.

A8: wires and clamps have been fitted.

A9: no operation errors for intermediate process have been confirmed, and report has been made.

A10: emergency repair task of overhead transmission lines has finished.

T1: pole was climbed.

T2: the voltage of transmission lines with electroscope has been checked.

T3: ground wire has been set up according to operating instruction.

T4: measures to prevent ground wire from swing have been taken.

T5: steel wire safety knots have been passed to electricians on transmission lines from the ground.

T6: conductors and clamps were lifted, and the used ones were replaced.

T7: bolts have been fastened, checking if there are tools left over and climbing down pole to report.

T8: the ground wire was removed.

B1: if ground wire is fixed, then the process goes to A7; otherwise, it goes to $\mathrm{T} 4$.

The first- and the second-order entropy of process control flowchart in emergency repair task are classified and listed in Table 2.

According to Section 2 in this paper, the quantified entropy value of complexity of each impact factor can be calculated. 


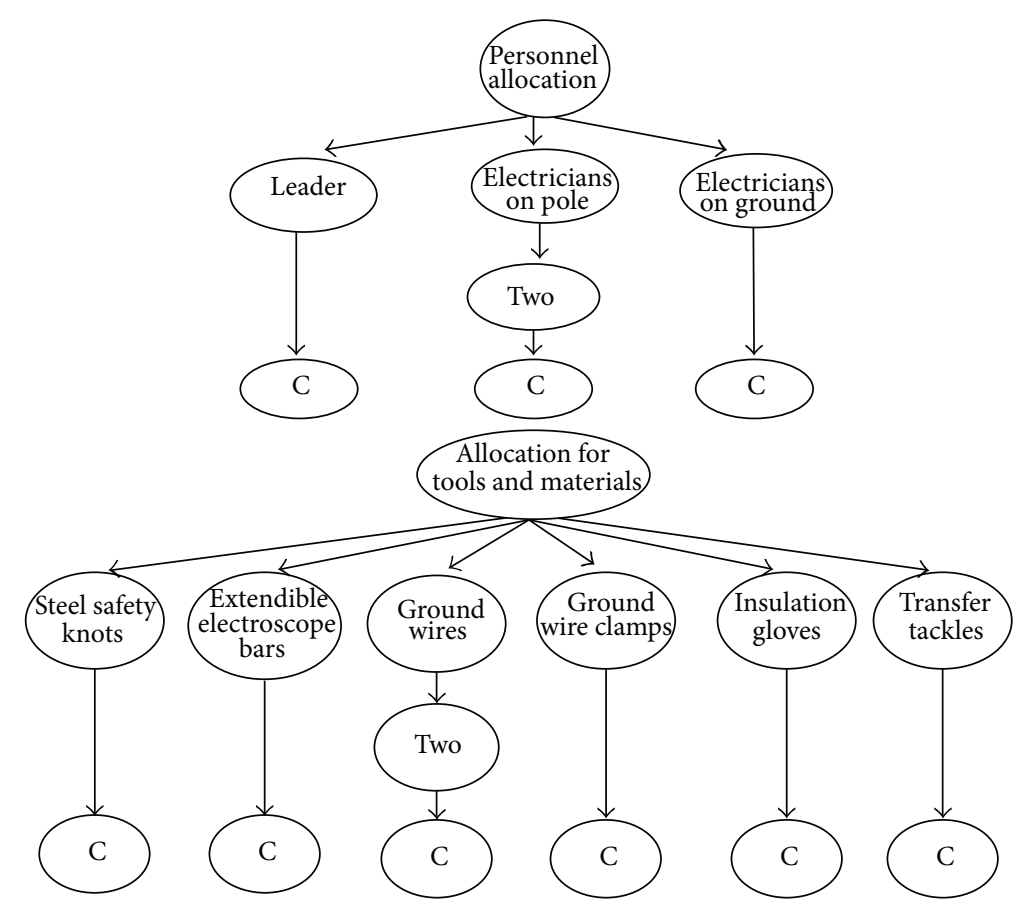

FIGURE 3: Information structure chart model for emergency repair task.
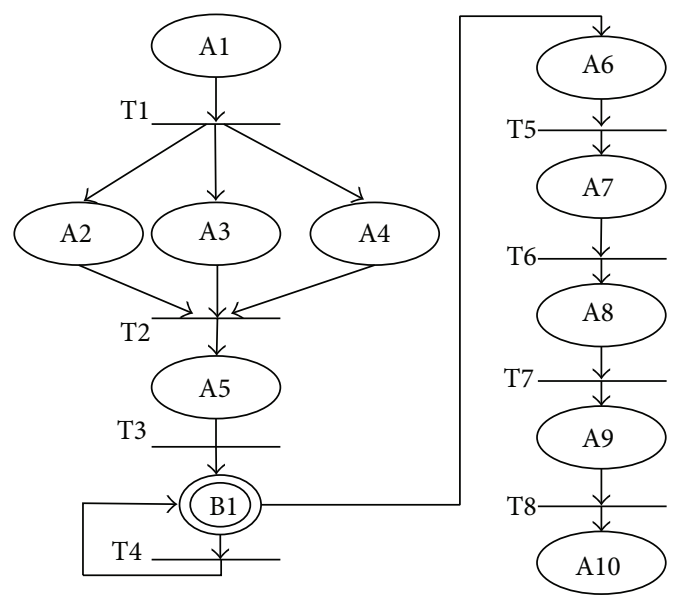

FIGURE 4: Process control flowchart model for emergency repair of overhead transmission lines.

The second-order entropy of information structure chart for emergency repair task is as below:

$$
\begin{aligned}
G_{2} & =-\sum_{i=1}^{21} p\left(A_{i}\right) \log _{2} p\left(A_{i}\right) \\
& =-20\left(\frac{1}{22} \log _{2} \frac{1}{22}\right)-\left(\frac{2}{22} \log _{2} \frac{2}{22}\right) \\
& =4.3685 .
\end{aligned}
$$

The first-order entropy of process control flowchart for emergency repair task is as below:

$$
\begin{aligned}
G_{1}^{\prime} & =-\sum_{i=1}^{6} p\left(A_{i}\right) \log _{2} p\left(A_{i}\right) \\
& =-\left(\frac{14}{19} \log _{2} \frac{14}{19}\right)-5\left(\frac{1}{19} \log _{2} \frac{1}{19}\right)=1.4425 .
\end{aligned}
$$

The second-order entropy of process control flowchart for emergency repair task is as below:

$$
\begin{aligned}
G_{2}^{\prime} & =-\sum_{i=1}^{17} p\left(A_{i}\right) \log _{2} p\left(A_{i}\right) \\
& =-16\left(\frac{1}{19} \log _{2} \frac{1}{19}\right)-\left(\frac{3}{19} \log _{2} \frac{3}{19}\right)=3.9977 .
\end{aligned}
$$

According to the three impact factors on the complexity of emergency repair task of overhead transmission line, experts' judgment matrixes are here below:

$$
\begin{gathered}
A^{1}=\left[\begin{array}{ccc}
1 & 4 & 2 \\
0.25 & 1 & 0.6 \\
0.5 & 1.67 & 1
\end{array}\right] \quad A^{2}=\left[\begin{array}{ccc}
1 & 3 & 0.75 \\
0.33 & 1 & 0.3 \\
1.33 & 3.33 & 1
\end{array}\right], \\
A^{3}=\left[\begin{array}{ccc}
1 & 2 & 0.5 \\
0.5 & 1 & 0.35 \\
2 & 2.857 & 1
\end{array}\right] .
\end{gathered}
$$

The results of consistency test are $0.004135,-0.00058$, and 0.012115 , respectively, less than 0.1 , and all meet the criterion. The similarities are worked out as $c_{12}=0.912512$, 
TABLE 3: Quantified entropy values of task complexity and actual consumed time.

\begin{tabular}{|c|c|c|c|c|c|c|}
\hline \multirow{2}{*}{$\begin{array}{l}\text { Emergency repair } \\
\text { task }\end{array}$} & \multirow{2}{*}{ Information complexity } & \multirow{2}{*}{ Logic complexity } & \multirow{2}{*}{ Scale complexity } & \multicolumn{2}{|c|}{ Complexity of task } & \multirow{2}{*}{ Time (s) } \\
\hline & & & & Average weighting & AHP weighting & \\
\hline 1 & 3.5785 & 1.8456 & 3.5412 & 3.094255 & 2.710724 & 600 \\
\hline 2 & 4.5412 & 2.2145 & 2.4125 & 3.230857 & 2.950491 & 480 \\
\hline 3 & 5.5452 & 3.2451 & 6.1421 & 5.129207 & 4.525085 & 1530 \\
\hline 4 & 4.8542 & 2.2541 & 3.5745 & 3.713931 & 3.291971 & 630 \\
\hline 5 & 3.5874 & 1.5418 & 2.5412 & 2.688406 & 2.364063 & 350 \\
\hline 6 & 4.1254 & 2.5463 & 3.1247 & 3.328316 & 3.090249 & 310 \\
\hline 7 & 5.4214 & 3.2541 & 4.1286 & 4.357716 & 4.0222 & 850 \\
\hline 8 & 2.9548 & 1.1217 & 2.1589 & 2.208714 & 1.908412 & 240 \\
\hline 9 & 1.8574 & 0.9845 & 2.3154 & 1.804667 & 1.547446 & 210 \\
\hline 10 & 4.5848 & 3.2579 & 6.1547 & 4.811276 & 4.279221 & 1108 \\
\hline 11 & 5.7895 & 2.5894 & 6.8744 & 5.39732 & 4.557066 & 1800 \\
\hline 12 & 5.2154 & 3.2154 & 6.4121 & 5.117798 & 4.495189 & 1400 \\
\hline 13 & 4.1154 & 3.2145 & 4.2154 & 3.872729 & 3.646977 & 670 \\
\hline 14 & 2.1551 & 0.5114 & 0.9511 & 1.391015 & 1.181877 & 190 \\
\hline 15 & 6.1541 & 2.1152 & 4.1548 & 4.455325 & 3.828616 & 1210 \\
\hline 16 & 3.2145 & 0.3251 & 0.8541 & 1.928473 & 1.607629 & 300 \\
\hline 17 & 5.2148 & 2.2151 & 7.5412 & 5.443085 & 4.488074 & 1250 \\
\hline 18 & 1.1218 & 0.3241 & 0.5214 & 0.737947 & 0.635086 & 150 \\
\hline 19 & 5.1241 & 3.2145 & 4.2152 & 4.254526 & 3.930456 & 780 \\
\hline 20 & 0.8115 & 0.2154 & 0.5215 & 0.570355 & 0.480978 & 145 \\
\hline
\end{tabular}

$c_{23}=0.986333$, and $c_{13}=0.825489$, respectively; that is, the second expert and the third expert are combined into one category. Therefore, the weights are $0.2,0.4$, and 0.4 for each type of experts. According to formula (8), weight coefficients $\alpha, \beta$, and $\lambda$ are calculated as $0.230515,0.575143$, and 0.194341 , respectively, for complexities of three impact factors. Based on formula (4), the quantified value of task's complexity is computed as below:

$$
\begin{aligned}
& \text { CT } \\
& =\sqrt{0.23 \times(4.37)^{2}+0.58 \times(1.44)^{2}+0.19 \times(4.00)^{2}} \\
& =8.6350 .
\end{aligned}
$$

In order to test the validity of complexity quantification based on AHP weighting method, average weighting method is used for comparison, and complexities in 20 emergency repair tasks are worked out by these two methods as shown in Table 3. The actual consumed time of emergency operation is set based on the historical statistic data from maintenance stations.

Nonlinear curve fitting within a 95\% prediction interval is conducted with data in Table 3 , and the comparison of accuracy for these two methods is made as shown in Figures 5 and 6. In Figure 5, the relationship between average weighting value of complexity quantification and the consumed time

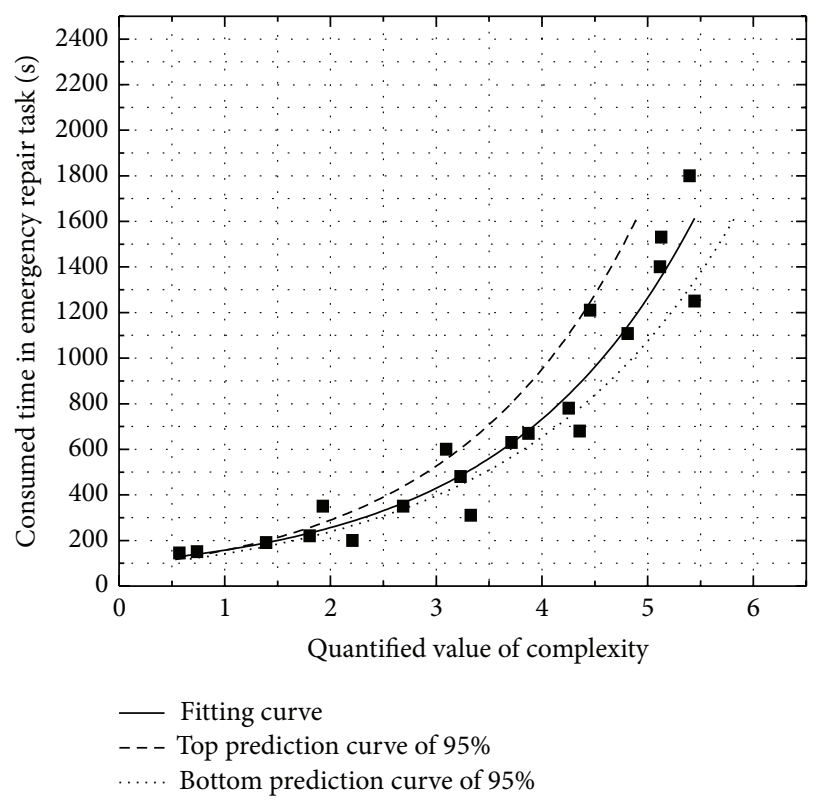

FIGURE 5: Complexity quantification based on average weighting.

in emergency operation is illustrated. In Figure 6, the relationship between AHP weighting value of complexity quantification and the consumed time in emergency operation is shown. 


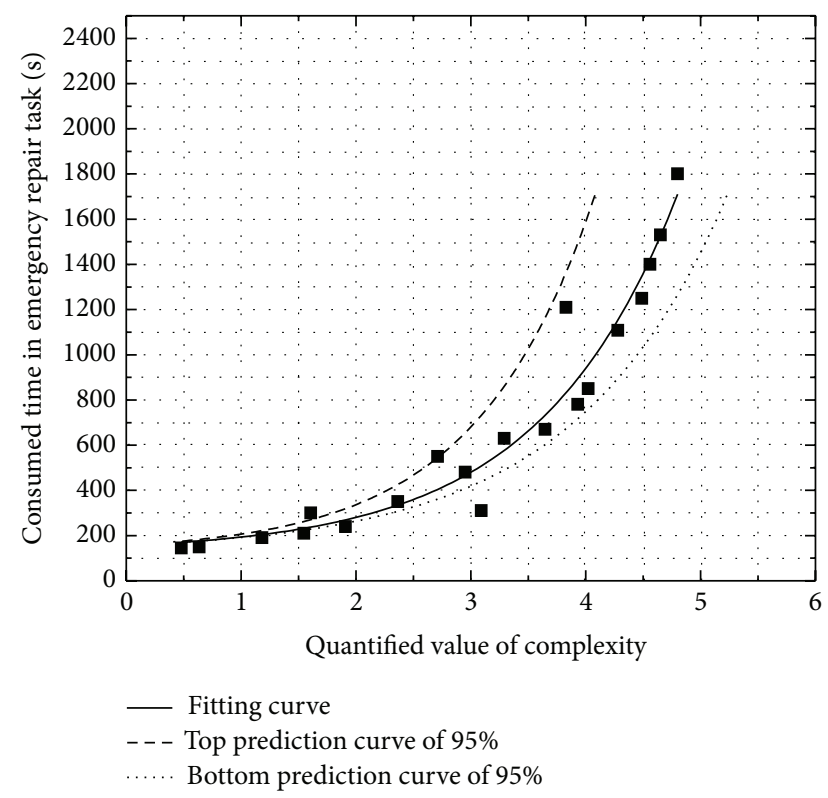

FIGURE 6: Complexity quantification based on group AHP weighting.

The fitting curve in Figure 6 shows a more significant trend of synchrony to increase or decrease between the quantitative value of TC (task complexity) and the consumed time in emergency repair task than the one in Figure 5. Therefore, AHP weighting method can reflect the complexity as well as the workload in emergency repair task. By comparing scatterplots in the two figures, points in Figure 6 distribute more intensively within a $95 \%$ prediction interval than those in Figure 5, which proves that AHP weighting method is more preferred to average weighting method for complexity quantification of task.

\section{Conclusion}

The implementation of emergency repair scheme can prevent the accident from further deterioration [16], and the process to execute the emergency repair scheme is just the process to finish the emergency repair task; therefore, complexity of task will directly influence the smooth degree while implementing the emergency repair scheme.

Petri net is used as graphical modeling for whole emergency repair scheme to fully demonstrate information on personnel and tools and the logic of process which influence the complexity of tasks; therefore, the accuracy of quantified values for various factors based on graph entropy method has been greatly improved.

In the process of quantifying the overall complexity, an improvement has been done; that is, experts' advice on the relative importance of impact factors to emergency repair task of overhead transmission lines is taken into account by group AHP method while setting weights. This improvement not only makes a much more professional design of weighting, but also helps to decrease the subjective errors greatly. By comparing with average weighting method, group
AHP weighting method shows a better performance and its validity is also proved by the trend of synchrony to increase or decrease between the quantified value of task complexity and the consumed time in emergency repair task.

\section{Conflict of Interests}

The authors declare that there is no conflict of interests regarding the publication of this paper.

\section{Acknowledgments}

This paper is supported by Beijing Higher Education Young Elite Teacher Project (Grant no. YETP0704) and the Science \& Technology Key Project of State Grid Corporation of China (no. SGSX0000AZJS1400173).

\section{References}

[1] S. Ekici, "Support Vector Machines for classification and locating faults on transmission lines," Applied Soft Computing Journal, vol. 12, no. 6, pp. 1650-1658, 2012.

[2] Z. Jing, L. Cunbin, and M. Weiqing, "Path planning for urgent repair of transmission line based on diamond-boundary limit algorithm," Information Technology Journal, vol. 12, no. 22, pp. 6852-6858, 2013.

[3] D. Mendonca, G. E. G. Beroggi, and W. A. Wallace, "Evaluating support for improvisation in simulated emergency scenarios," in Proceedings of the 36th Hawaii International Conference on Systems Sciences, Big Island, Hawaii, USA, January 2003.

[4] J. Park and W. Jung, "A study on the development of a task complexity measure for emergency operating procedures of nuclear power plants," Reliability Engineering and System Safety, vol. 92, no. 8, pp. 1102-1116, 2007.

[5] S. Li, Q. Cai, and B. Zhu, "Research on the quantification of emergency Maintenance Task Complexity for marine power facility," Journal of Wuhan University of Technology (Transportation Science and Engineering), vol. 34, no. 4, pp. 813-817, 2010.

[6] Z. F. You, Q. Shi, Q. W. Hu, and Y. Wang, "A Study on quantification model for BDR scheme complexity based on diagram entropy methodology," Journal of Ordnance Engineering College, vol. 25, no. 2, pp. 7-11, 2013.

[7] J. Park, W. Jung, and J. Ha, "Development of the step complexity measure for emergency operating procedures using entropy concepts," Reliability Engineering and System Safety, vol. 71, no. 2, pp. 115-130, 2001.

[8] M. Dehmer and A. Mowshowitz, "A history of graph entropy measures," Information Sciences, vol. 181, no. 1, pp. 57-78, 2011.

[9] J. B. He, Q. X. Su, and H. Q. Gu, "Virtual maintenance process model based on extended Petri net," Computer Simulation, vol. 27, no. 3, pp. 254-257, 2010.

[10] H. Gomaa, Software Modeling and Design: UML, Use Cases, Patterns, and Software Architectures, Cambridge University Press, Cambridge, UK, 2011.

[11] G. Morison, Z. Tieges, and K. Kilborn, "Analysis of electroencephalography activity in early stage alzheimer's disease using a multiscale statistical complexity measure," Advanced Science Letters, vol. 19, no. 8, pp. 2414-2418, 2013.

[12] Z. Z. Zhang, M. L. Littman, and X. P. Chen, "Covering number as a complexity measure for POMDP planning and learning," in 
Proceedings of the 26th AAAI Conference on Artificial Intelligence and the 24th Innovative Applications of Artificial Intelligence Conference, pp. 1853-1859, Toronto, Canada, July 2012.

[13] R. Zhang, "Knowledge management strategy implementation performance evaluation based on BSC," Electrotehnica, Electronica, Automatica, vol. 61, no. 3, pp. 81-87, 2013.

[14] Y. Y. Wu, Z. S. Hua, and Y. Zha, "Calculation of the weights and the amalgamation of the matrixes in AHP group decision," Operations Research and Management Science, vol. 12, no. 4, pp. 16-21, 2003.

[15] L. F. Wang and S. B. Xu, AHP Introduction, China Renmin University Press, Beijing, China, 1990.

[16] A. M. Ifrim, "Quality assurance in project management," Electrotehnica, Electronica, Automatica, vol. 61, no. 2, pp. 79-84, 2003. 


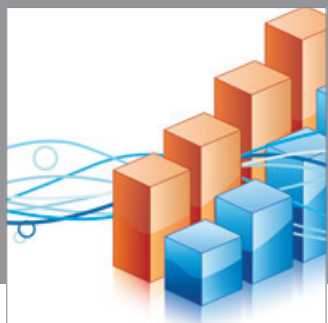

Advances in

Operations Research

mansans

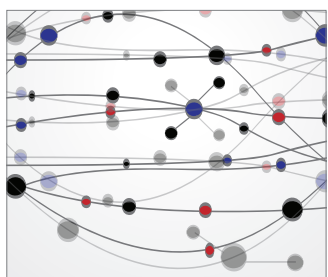

The Scientific World Journal
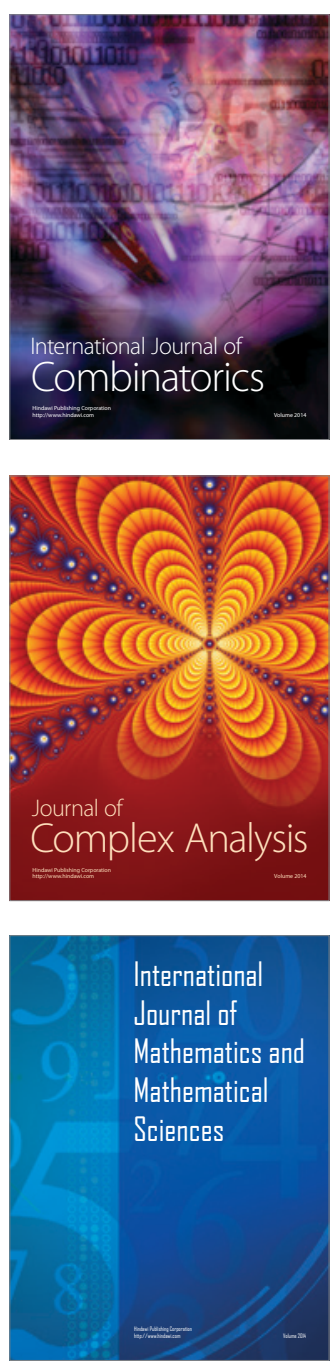
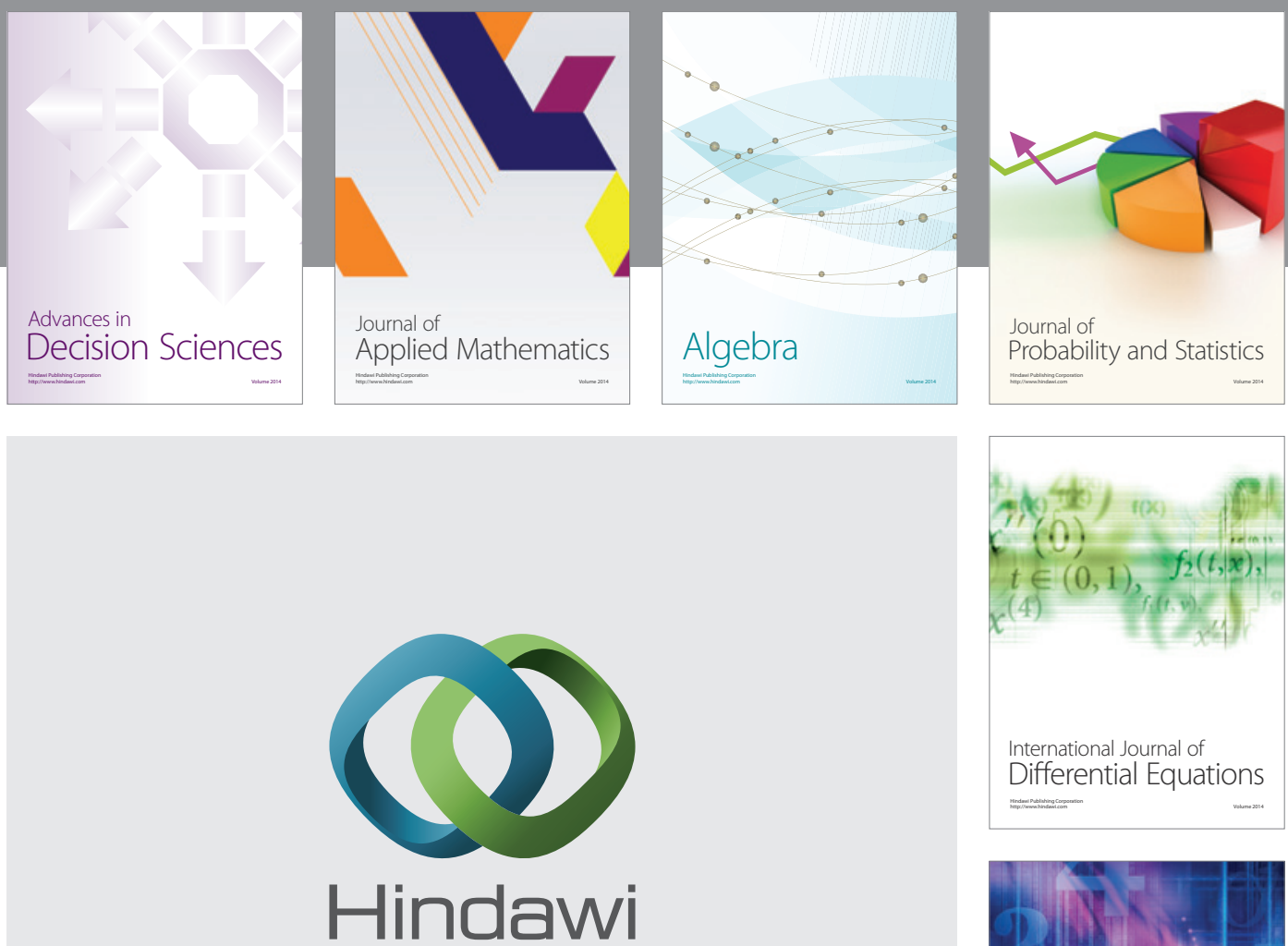

Submit your manuscripts at http://www.hindawi.com
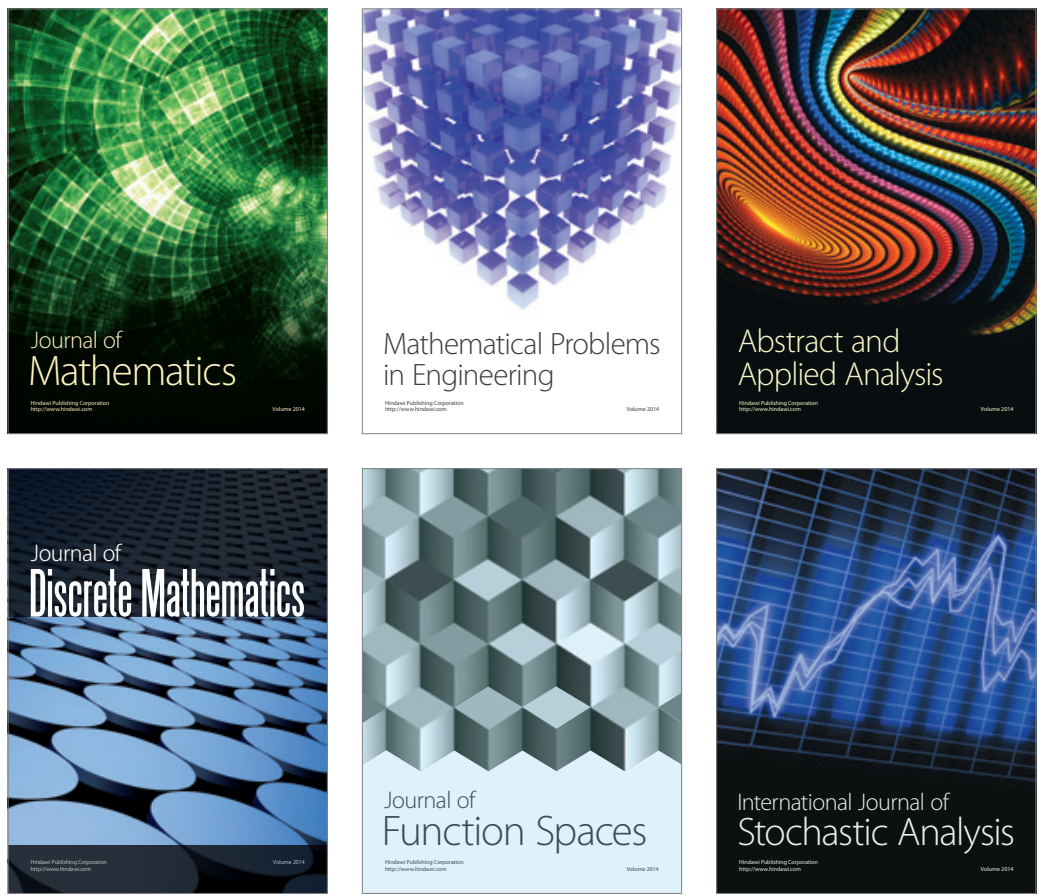

Journal of

Function Spaces

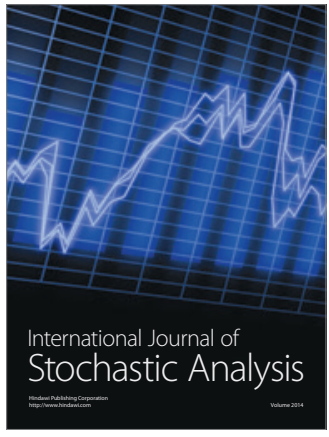

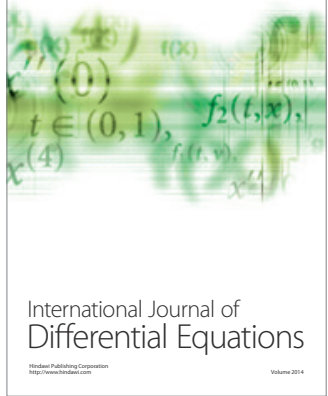
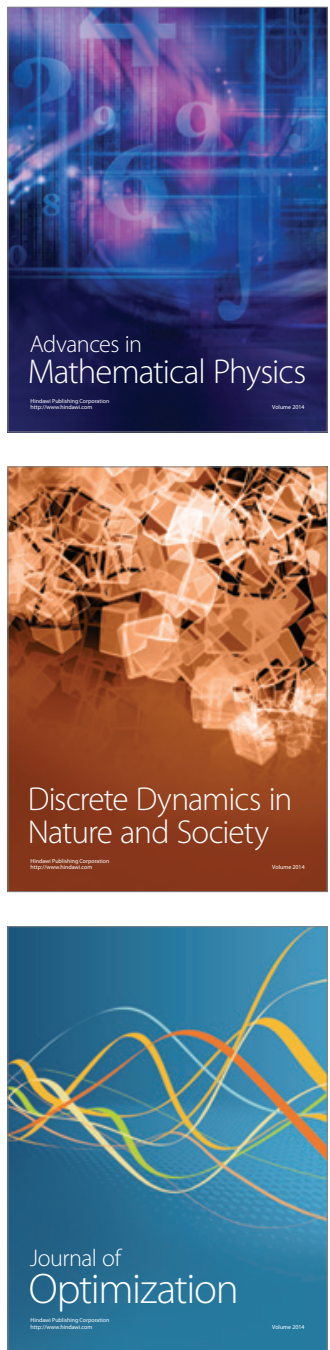